\title{
Insilco binding analysis of MKP-1 inhibitors
}

\author{
Sukesh $\mathrm{K}^{1 *}$, Lavanya $\mathrm{C}^{2}$ and Balavinayagamani $\mathrm{G}^{2}$ \\ ${ }^{1}$ Department of Bioinformatics, Faculty of Engineering \& Technology, School of Bioengineering, SRM \\ University, Kattankulathur, 603203,Kancheepuram, Tamil Nadu, India \\ ${ }^{2}$ Department of Bioinformatics, SRM Arts and Science College, Kattankulathur, 603203, Kancheepuram, \\ Tamil Nadu, India, sukesh1411@gmail.com
}

\begin{abstract}
Small cell-permeant inhibitors of protein kinases have become valuable reagents to investigate the physiological roles of protein kinases, because they can be used simply and rapidly to block endogenous kinase activity in normal cells and tissues, as well as transformed cell lines. There are no potent inhibitors of MKP-1, which dephosphorylates p38, JNK, and this is unfortunate because MKP-1 has been associated with human neoplasia and is an attractive potential therapeutic target. The active sites of MKP-1 were predicted and screened with synthesized analogs and related structures are obtained from PUBCHEM. The best hits were the analogs NU-126, NU-154 and these compounds were docked with the protein using AUTODOCK. MKP-1 constitutes an exciting, novel potential therapeutic target for Rheumatoid Arthritis. There is perfect active site information about the protein and selective inhibitor, so this docking work provides insights to develop new drug for the disease, rheumatoid arthritis.

KEYWORDS: Rheumatoid arthritis, MKP-1 inhibitors, protein kinases, synthesized analogs, docking, active
\end{abstract} site.

\section{Introduction \\ Protein Kinases}

The human genome contains about 520 protein kinase genes which constitute about $2 \%$ of all eukaryotic genes. The chemical activity of a kinase involves removing a phosphate group from ATP and covalently attaching it to one of three amino acids that have a free hydroxyl group. Most kinases act on both serine and threonine; others act on tyrosine, and a few (dual specificity kinases) act on all three [1].

\section{Human Mitogen Activated Protein Kinase Phosphatase 1 \\ The protein tyrosine phosphatases (PTPs) constitute a family of closely related key regulatory enzymes that dephosphorylates phosphotyrosine residues in their protein substrates. Malfunctions in PTP activity are linked to various diseases. Consequently, PTPs have emerged as promising targets for therapeutic intervention in recent years [2]. Protein kinases and phosphatases are likely targets for the development of therapeutic drugs since they are involved in specific signaling pathways which regulate cell functions such as metabolism, cell cycle progression, cell adhesion, vascular function and angiogenesis [3]. Mitogen- activated protein kinase phosphatase-1 (MKP-1) play crucial roles in cellular signaling in response to signals like mitogenesis, programmed cell death, differentiation, and secretion of cytokines. Pac-1 and MKP-1 have specificity for MAP kinases [4]. MKP 1 is a member of the MAP kinase family. MAP kinases, also known as extracellular signal-regulated kinases (ERKs), act as an integration point for multiple biochemical signals, and are involved in a wide variety of cellular processes such as proliferation, differentiation, transcription regulation and}

development [5]. The activation of this kinase requires its phosphorylation by upstream kinases. Upon activation, this kinase translocates to the nucleus of the stimulated cells, where it phosphorylates nuclear targets. Two alternatively spliced transcript variants encoding the same protein, but differing in the UTRs, have been reported for this gene. Mitogen-activated protein kinase phosphatase-1 (MKP-1 or DUSP1) dephosphorylates and inactivates mitogenactivated protein kinase (MAPK) substrates, such as p38, JNK [6]. There are no potent inhibitors of MKP-1, which dephosphorylates p38, JNK, and this is unfortunate because MKP-1 has been associated with human neoplasia and is an attractive potential therapeutic target. The lack of readily available selective small molecule inhibitors of MKP family members has severely limited interrogation of their biological role. A small molecule inhibitor of MKP-3, namely NSC 357756 (6-(4,5-dihydro-1H-imidazol- 2-yl)-2-(2-[5(4,5-dihydro-1H-imidazol-2-yl)-benzofuran-2-yl]vinyl)-1 $\mathrm{H}$-indol-3-ylamine)is justified because human MKP-3 shares 36\% amino acid identity with human MKP-1 but the 11 amino acid catalytic domain is $82 \%$ identical [7]. NSC 357756 is a previously identified small molecule inhibitor of MKP-3. NSC 357756 has also been reported to inhibit a Zn-dependent metalloprotease, namely anthrax lethal factor protease, which decreases the phosphorylation and activation of extracellular signal-regulated kinases. The properties of NSC 357756 have been mentioned in the Table 1 [7]. NSC 357756 is a dual specificity phosphatase inhibitor. A dual specificity phosphatase sub type that play role in intracellular signal transduction by inactivating mitogen-activated protein kinases. It has specificity for extra cellular signal-regulated map 
kinases [7]. From the previously identified inhibitor (NSC 357756) of MKP-3, seven NSC 357756 congeners are synthesized, which were evaluated for in vitro inhibition against several protein phosphatases. The newly synthesized analog NU-126 [2-((E)-2-(5-cyanobenzofuran-2yl) vinyl)- $1 \mathrm{H}$-indole-6-carbonitrile] inhibited MKP1 and VHR in vitro but was less active against human MKP-3, Cdc25B, and PTP1B. The inhibition of MKP-1 by NU-126 was independent of redox processes. The chemical structures of NSC 357756 and newly synthesized analogs have been mentioned in Figure 1 [7]. NU-145, which is a substructure of NU-126, NU-176, and NU2-157, which lacked the benzofuran moiety [7]. Surprisingly, we found no potent in vitro inhibitors of MKP-3, although NU2-168 and NU2157 inhibited MKP-3 with IC50 values of 73.3 and $70.0 \mathrm{IM}$, respectively. The bis-nitrile NU-126, however, inhibited the dual specificity phosphatases MKP-1 and VHR with IC50 values of $28.8 \pm 2.9 \mathrm{IM}$ and $38.1 \pm 2.8 \mathrm{IM}$; at $100 \mathrm{IM} \mathrm{NU}$ 126 inhibited PTP1B by only $30 \%$ and at $400 \mathrm{IM}$ it inhibited Cdc25B and MKP-3 by $30-40 \%$. Rheumatoid arthritis (RA) is a chronic autoimmune disease in which imbalances in proand anti-inflammatory cytokines promote the induction of autoimmunity, inflammation and joint destruction. The importance of inflammatory cytokines in the pathogenesis of RA has been underscored by the success of biologics that act to block the effects of cytokines, such as tumour necrosis factor- , interleukin (IL)- 1 or IL-6, in treating disease. Mitogen-activated protein kinases (MAPKs) have been implicated as playing key regulatory roles in the production of these pro-inflammatory cytokines and downstream signalling events leading to joint inflammation and destruction. Hence Mitogen activated protein kinase phosphatase (MKP-1) has become a novel target for Rheumatoid Arthritis [8].

\section{Methodology}

\section{Ligand Structure Preparation}

Initially the hydrogens were added to all the atoms in the ligand and ensured that their valences were completed. This was done using this molecular modeling package (ADT). It was made sure that the atom types were correct before adding hydrogens. Depending on whether charged or neutral carboxylates and amides are desired the PH was specified automatically. Next, partial atomic charges were assigned to the ligand molecule. AMPAC or MOPAC was used to generate partial atomic charges for the ligand. These charges were written in 'pdbqs' format, which had the same columns as a Brookhaven PDB format, but with an added column of partial atomic charges.

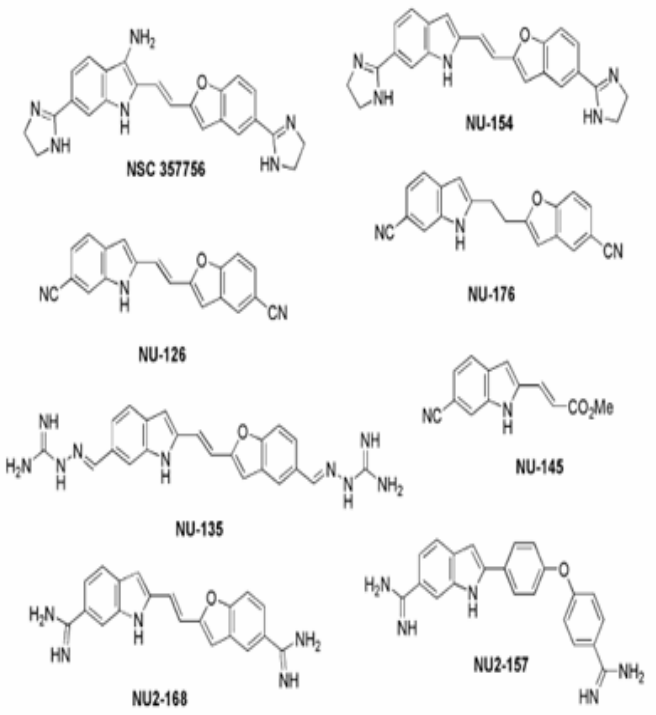

Fig. 1- The chemical structures of NSC 357756 and newly synthesized analogs (source ref. 7)

\section{Ligand Flexibility}

To allow flexibility in the ligand, the rotatable bonds were assigned. A plot of the ligand, labeled by atom name, and a second labeled by atom serial number (atom ID). AutoDock can handle up to MAX_TORS rotatable bonds: this parameter is defined in "autodock.h", and is ordinarily set to 32 . If this value is changed, AutoDock must be recompiled.

\section{Protein Structure Preparation}

When modeling hydrogen bonds, polar hydrogens were added to the protein. Then the appropriate partial atomic charges were assigned. The charged proteins were converted to 'pdbqs' format so that AutoGrid can read it. It was noted that in most modeling systems polar hydrogens were added in a default orientation, assuming each new torsion angle was $0^{\circ}$ or $180^{\circ}$. Without some form of refinement, this would lead to spurious locations for hydrogen-bonds. One option is that the hydrogens were relaxed and a molecular mechanics minimization was performed on the structure. Another one was that a program like "pol_h" was used where the default-added polar hydrogen structure, was taken as input favorable locations for each movable proton, were sampled and the best position for each was selected. This "intelligent" placement of movable polar hydrogens would be particularly important for tyrosines, serines and threonine.

\section{Autogrid \\ Grid Maps}

The pre-calculated grid maps, one for each atom type present in the ligand being docked were required for Autodock to make the docking 
calculations extremely fast. These maps were calculated by AutoGrid. A grid map was created with a three dimensional lattice of regularly spaced points, surrounding (either entirely or partly) and centered on the active site of the macromolecule .Typical grid point spacing varies from $0.2 \AA$ to $1.0 \AA$, although the default was $0.375 \AA$ (roughly a quarter of the length of a carbon-carbon single bond). The potential energy of a 'probe' atom or functional group that is due to all the atoms in the macromolecule was stored in each point with in the grid map. A even number of grid points in each dimension, $\mathrm{nx}$, ny and $\mathrm{n}$ was specified as AutoGrid adds a central point and AutoDock requires an odd number of grid points.

Running Auto Grid

An input grid parameter file, which usually has the extension ".gpf" was required for Autogrid. The maximum and minimum energies found during the grid calculations were given in the log file. The grid maps were written in ASCII form by Autogrid, for readability and portability; AutoDock reads ASCII format grid maps.

\section{Autodock}

Running AutoDock

Once the grid maps have been prepared by AutoGrid and the docking parameter file, or 'dpf', is ready, the user is ready to run an AutoDock job. The docking results were viewed using "getdocked", a PDB formatted file was created. It was called "lig.macro.dlg.pdb" and will contain all the docked conformations output by AutoDock in the "lig.macro.dlg" file.

\section{Docking Runs}

The target protein was taken; water molecules and ions were removed from the protein. The proteins was then modified and changed to a desired format (pdbqs) using ADT.

A. Ligand Preparation

The ligand was obtained from literature reference and designed using chemsketch [9].

B. Protein Preparation

A 'pdbqs' file was prepared by adding the following parameters to the PDB file.

1. Kollmann Charges.

2. Solvation parameters.

\section{Docking}

Docking was performed using AutoDock. The following parameters were used,

Number of runs: 50 .

Grid Size: 54 x 50 x 40 .

Cell spacing: 0.375 .

Grid Centre: $x=3.073$

$$
\begin{aligned}
& y=-5.088 \\
& z=-8.057
\end{aligned}
$$

\section{Results and Discussion Active Site Prediction Performed Using SPDBV}

There are no active sites for the Mitogen Activated Protein Kinase Phosphatase 1 in PDB as it is not a complex protein. Hence the active sites were predicted using the Swiss PDB Viewer [10]. The active sites were Gln 228, Met 229, Ile 232, Ile261, Arg 263, and Thr266 shown in figure 2.

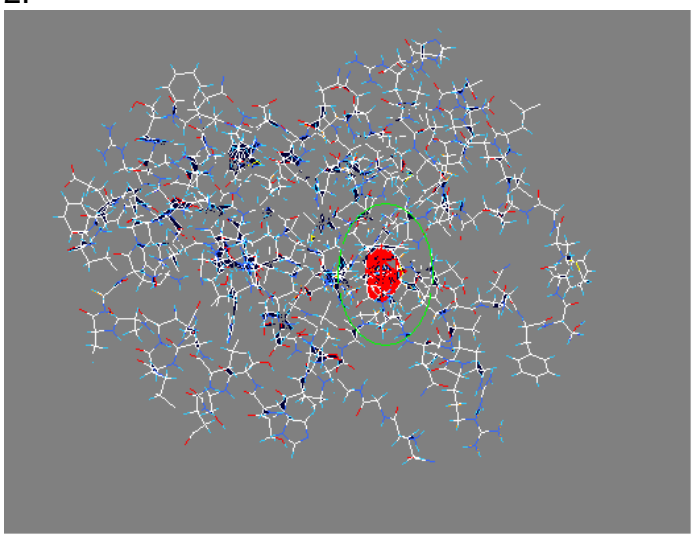

Fig. 2- Cavity showing the active site region

\section{Virtual Screening Performed Using Argus Lab:}

The newly synthesized analogs from the inhibitor NSC 357756 including NU-154, NU-135, NU2157, NU2-168, NU-126, NU-176, NU-145 and its chemically related structures(CID:5771685, 5458834, 10023723, 5478778, 10180044, 5478780, 435423, 5478783, 18476216, 5478786, 5478777, 435425, 52600, 439117)are retrieved from the PDBCHEM and further screened using the ARGUS LAB [11].

The screened result was given below.

Summary of results in order of docking score (kcal/mol)

$\begin{array}{ll}\text { 1. NU-126 } & -10.6342 \\ \text { 2. NU-154 } & -10.4841 \\ \text { 3. NU-176 } & -9.75016 \\ \text { 4. NU-145 } & -9.37547 \\ \text { 5. } 18476216 & -6.26591\end{array}$

The synthesized analog $\mathrm{NU}-126$ is the best docked compound with the score -10.6342 $\mathrm{kcal} / \mathrm{mol}$ and also the analog NU-154 shows the docking score -10.4841. As mentioned in the literature [7], The bis-nitrile NU-126, inhibited the dual specificity phosphatases MKP-1 with IC50 values of $28.8 \pm 2.9 \mu \mathrm{M}$ and NU-154 inhibited human PTP1B in vitro with an IC50 value of $24 \pm$ $1 \mu \mathrm{M}$ and showed little inhibition against Cdc25B, MKP-1, and VHR phosphatases. The compounds NU-126, NU-154 is further docked to the above protein with the AUTODOCK 3.0 [12]. 


\section{Validation Of Docking Results Using Autodock \\ The molecular docking was performed using} Genetic Algorithm - Least Square (GA-LS) algorithm optimized with autodock tool [12]. From the 50 poses of docking, the complex formed with least energy and with the top rank chosen as the stable conformation. The clustering histogram is collected from the docking log file.

Automated Docking of Ligand (NU-126) to Macromolecular Receptor:

\section{Clustering Histogram}

Genetic algorithm was performed for 50 runs .The dock log file shows that the ligand docked with the Mitogen activated protein kinase phosphatase 1 with the lowest energy -7.07 $\mathrm{kcal} / \mathrm{mol}$ (run 25) and shown rank 1 in the Clustering Histogram table. Interaction of NU-126 with MKP 1 shown in figure 3 , and the hydrogen bond donor, acceptor and distance profile are mentioned in table 2.

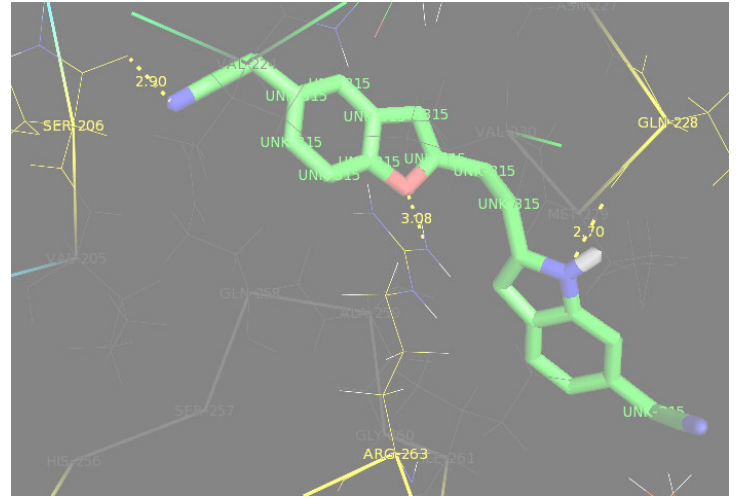

Fig. 3- Interaction of NU-126 with MKP 1

Finally, based on the outcomes of molecular docking process, the complex formed in run 25 is considered as stable complex as it shows the good interaction with the active site amino acids with least docked energy. Automated Docking of Ligand (NU-154) to Macromolecular Receptor From the 50 poses of docking, the complex formed with least energy and with the top rank chosen as the stable conformation. The clustering histogram is collected from docking log (dlg) file.

\section{Clustering Histogram}

Genetic algorithm was performed for 50 runs .The dock log file shows that the ligand docked with the Mitogen activated protein kinase phosphatase 1 with the lowest energy -8.28 $\mathrm{kcal} / \mathrm{mol}$ (run 8) and shown rank 1. Interaction of $\mathrm{NU}-126$ with MKP 1 shown in figure 4, and the hydrogen bond donor, acceptor and distance profile are mentioned in table 3.

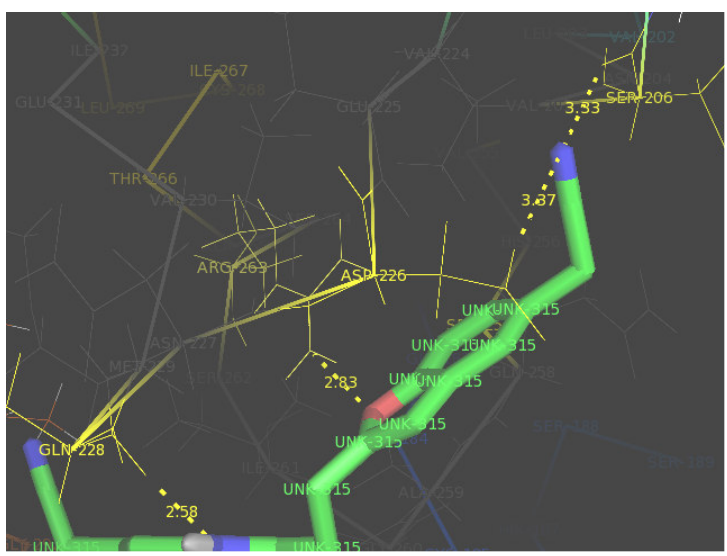

Fig.4- Interaction of NU-154 with MKP 1

Finally, based on the outcomes of molecular docking process, the complex formed in run 8 is considered as stable complex as it shows the good interaction with the active site amino acids with least docked energy. From the 50 poses of molecular docking, the complex 1 (MKP-1 with $\mathrm{NU}-126)$ formed with least energy $-7.07 \mathrm{kcal} / \mathrm{mol}$, complex 2 (MKP-1 with NU-154) formed with least energy $-8.28 \mathrm{kcal} / \mathrm{mol}$ and with the top rank chosen as the stable conformation. Finally, based on the outcomes of molecular docking process, the complex formed in run 25 and run 8 is considered as stable complex as it shows the good interaction with the active site amino acids with least docked energy. There is perfect active site information about the protein and selective inhibitor, so this work provides insights to develop new drug for the disease, rheumatoid arthritis. The scope extends to finding the biological activity of the compounds, which might be used as a candidate lead compounds for future studies.

\section{Conclusion}

The active sites of MKP-1 were predicted and screened with synthesized analogs and related structures obtained from PUBCHEM. The best hits were the analogs NU-126, NU-154 and these compounds were docked with the protein using AUTODOCK. MKP-1 constitutes an exciting, novel potential therapeutic target for Rheumatoid Arthritis. There is perfect active site information about the protein and selective inhibitor, so this Docking work provides insights to develop new drug for the disease, rheumatoid arthritis.

\section{References}

[1] Manning G., Whyte D.B., Martinez R., Hunter T., and Sudarsanam S. (2002) Science, 98(5600), 1912-34.

[2] Purushottam M., Dewang, Nai-Mu Hsu, Shao-Zheng Peng and Wen-Ren Li, (2005) Current Medicinal Chemistry, (12), 1-22 
[3] Sridhar R., Hanson-Painton O., and Cooper D.R. (2000) Pharma Res. 17(11), 134553.

[4] Ralph, Jennifer, Morand, Eric F. (2008) Informa Healthcare, 12(7), 795-808.

[5] Lee J.C., Laydon J.T., McDonnell P.C., Gallagher T.F., Kumar S., Green D., McNulty D., Blumenthal M.J., Heys J.R., Landvatter S.W., et al.(1994) Nature, 372(6508), 739-46.

[6] Hammer M., Mages J., Dietrich H., Servatius A., Howells N., Cato A. and Lang R. (2006) J. Exp. Med., 203, 1520.

[7] John S. Lazo, Ruth Nunesc, John J. Skokoa, Pierre E. Queiroz de Oliveiraa, Andreas Vogta, and Peter Wipf (2006) Bioorg Med Chem., 14, 5643-5650.

[8] Thalhamer T., McGrath M.A., Harnett M.M. (2008) Rheumatology, 4, 409-14.

[9] Gary O. Spessard (1998) J. Chem. Inf. Comput. Sci., 38(6), 1250-1253.

[10] Guex N., and Peitsch M.C. (1996) SwissPdbViewer: A Fast and Easy-to-use PDB Viewer for Macintosh, 77, 7

[11] Saju Joy, Parvathy S. Nair, Ramkumar Hariharan and M. Radhakrishna Pillai (2006) In Silico Biology, 6, 601-605

[12] Wang R., Wang Y., Lu S. (2003) J. Med. Chem., 46, 2287- 2303. 
Table 1- Properties Of NSC 357756.

\begin{tabular}{|l|c|}
\hline MOLECULAR WEIGHT & $445.924(\mathrm{~g} / \mathrm{mol})$ \\
\hline MOLECULAR FORMULA & C24H22C1N6O \\
\hline ROTATABLE BONDS & 4 \\
\hline H - BOND ACCEPTOR & 4 \\
\hline H - BOND DONOR & 8 \\
\hline
\end{tabular}

Table 2- Interaction and Binding Free Energy Of Complex 1 (NU-126 WITH MKP 1)

\begin{tabular}{|c|c|c|c|c|}
\hline \multirow[t]{2}{*}{ S.No } & \multirow[t]{2}{*}{ Interactions } & \multicolumn{2}{|c|}{ H- Bond } & \multirow[t]{2}{*}{ Distance $(\dot{A})$} \\
\hline & & Acceptor & Donor & \\
\hline 1 & $\mathrm{O}---\mathrm{HN}$ & $\mathrm{O}($ Ser 206) & HN(Ligand) & 2.90 \\
\hline 2 & $\mathrm{O}---\mathrm{HN}$ & $\mathrm{O}(\mathrm{Gln} 228)$ & HN(Ligand) & 2.70 \\
\hline 3 & O1 --- HN & O1(Ligand) & $\mathrm{HN}(\operatorname{Arg} 263)$ & 3.08 \\
\hline
\end{tabular}

Table 3- Interaction and Binding Free Energy of Complex 2 (NU-154 with MKP 1)

\begin{tabular}{|c|c|c|c|c|}
\hline \multirow[t]{2}{*}{ S.No } & \multirow[t]{2}{*}{ Interactions } & \multicolumn{2}{|c|}{ H- Bond } & \multirow{2}{*}{$\begin{array}{c}\text { Distance } \\
\text { (Å) }\end{array}$} \\
\hline & & Acceptor & Donor & \\
\hline$\overline{1}$ & $\mathrm{O}---\mathrm{HN}$ & $\mathrm{O}($ Ser 206) & $\mathrm{HN}$ (Ligand) & 3.33 \\
\hline 2 & $\mathrm{O}---\mathrm{HN}$ & $\mathrm{O}(\mathrm{Gln} 228)$ & $\mathrm{HN}$ (Ligand) & 2.58 \\
\hline 3 & OD1--- HN & OD1 (Asp 226) & $\mathrm{HN}$ (Ligand) & 3.37 \\
\hline 3 & O1--- HN & O1(Ligand) & $\mathrm{HN}(\operatorname{Arg} 263)$ & 2.83 \\
\hline
\end{tabular}

